\title{
Wavelengths, energy levels and hyperfine structure of Mn II and Sc II
}

\author{
G. Nave ${ }^{1}$, J. C. Pickering ${ }^{2}$, K. I. Townley-Smith ${ }^{3} \dagger$ and Hala ${ }^{4}$ \\ ${ }^{1}$ National Institute of Standards and Technology, Gaithersburg, MD, USA ${ }^{2}$ Imperial Colllege \\ London, London, UK ${ }^{3}$ Lamar University, Beaumont, TX, USA ${ }^{4}$ Aligarh Muslim University, \\ Aligarh, India
}

Abstract. We summarize our analysis of singly-ionized elements of astrophysical interest.

Keywords. atomic data

For many decades, the Atomic Spectroscopy Groups at the National Institute of Standards andTechnology (NIST) and Imperial College London (ICL) have measured atomic data of astronomical interest. Our spectrometers include Fourier transform (FT) spectrometers at NIST and ICL covering the region $1350 \AA$ to $5.5 \mu \mathrm{m}$ and a $10.7-\mathrm{m}$ grating spectrometer at NIST covering wavelengths from $300 \AA$ to $5000 \AA$. Sources for these spectra include high-current continuous and pulsed hollow cathode (HCL) lamps, Penning discharges, and sliding spark discharges. Recent work has focused on the measurement and analysis of wavelengths, energy levels, and hyperfine structure (HFS) constants for iron-group elements. The analysis of FT spectra of Cr I, Mn I, and Mn II is being led by ICL and is described in a companion paper (Pickering et al. 2015, this volume). Current work being led by NIST includes the analysis of HFS in Mn II, analysis of Mn II in the vacuum ultraviolet, and a comprehensive analysis of Sc II.

Comprehensive HFS constants for Mn II are needed for the interpretation of stellar spectra, and incorrect abundances may be obtained, when HFS is omitted. Holt et al. (1999) have measured HFS constants for 59 levels of Mn II using laser spectroscopy. We used FT spectra of $\mathrm{Mn} / \mathrm{Ni}$ and Mn/Cu HCLs covering wavelength ranges from $1350 \AA$ to $5.4 \mu \mathrm{m}$ to confirm 20 of the A constants of Holt et al. (1999) and obtain values for 47 additional levels.

Spectra of Sc HCLs have been recorded from $1800 \AA$ to $2.5 \mu \mathrm{m}$ using FT spectrometry at NIST. Additional measurements to cover wavelengths below $1800 \AA$ and above $2.5 \mu \mathrm{m}$ are in progress. The spectra are being analyzed to derive improved wavelengths, energy levels, and hyperfine structure parameters.

This work was partially supported by NASA, the STFC(UK), the Royal Society of the UK, and the Leverhulme Trust.

\section{References}

Pickering, J. C., Liggins, F., Clear, C., Ruffoni, M., Nave, G., \& Sansonetti, C. J. 2015, this volume

Holt, R. A., Scholl, T. J., \& Rosner, S. D. 1999, MNRAS, 306, 107

$\dagger$ NIST Summer Undergraduate Research Fellow. 